\title{
Metabolic syndrome and risk factors for cardiovascular disease: are nonagenarians protected?
}

\author{
Madlyn I. Frisard • Jennifer C. Rood • \\ Xiaobing Fang • Joseph Su • David A. Welsh • \\ S. Michal Jazwinski • Eric Ravussin • \\ for the Louisiana Healthy Aging Study
}

Received: 2 June 2008 / Accepted: 9 December 2008 / Published online: 31 January 2009

(C) The Author(s) 2009. This article is published with open access at Springerlink.com

\begin{abstract}
This study assessed cardiovascular disease risk factors in three groups of human subjects aged 20-34 [young, 20 male (M)/33 female (F)], 60-74 (aged, 29M/29F), and > 90 years (nonagenarian, $47 \mathrm{M} / 50 \mathrm{~F})$. Components of the metabolic syndrome, cardiovascular disease, and markers of inflammation and oxidative stress were assessed. Nonagenarians weighed less than the two other groups $(P<0.001)$; however, there was no difference in percent fat among the three groups. Aged individuals had the highest prevalence of the metabolic syndrome $(P<0.001)$ according to the Adult Treatment Panel III classification. Both fibrinogen and homocysteine concentra-
\end{abstract}

Louisiana Healthy Aging Study Meghan Allen, Arturo M. Arce, Mark A. Batzer, Lauri O. Byerley, Pauline Callinan, Cathy M. Champagne, Katie E. Cherry, Yu-wen Chiu, James P. DeLany, Melissa J. deVeer, Devon A. Dobrosielski, Andrea Ermolao, Elizabeth T. Fontham, Paula J. Geiselman, Valentina Greco, Sibte Hadi, Tiffany Hall, Karri Hawley, Scott W. Herke, Hui-Chen Hsu, Sangkyu Kim, Beth Kimball, Christina KingRowley, Kim Landry, Li Li, Hui-Yi Lin, Kay Lopez, John D. Mountz, Emily Olinde, Kim Pedersen, Henry Rothschild, Ryan A. Russell, Donald Scott, Jennie Silva, Nicole Standberry, Jessica Thomson, Crystal Traylor, Cruz Velasco-Gonzalez, Jerilyn A. Walker, Xui Yun Wang, Michael A. Welsch, Robert H. Wood, Pili Zhang.

Support This research was supported by the Louisiana Board of Regents through the Millennium Trust Health Excellence Fund [HEF(2001-06)-02], by the National Institute on Aging (P01AG022064), and by the National Institute of General Medical Sciences (GM42056 and GM15431). tions were significantly higher in the nonagenarians compared to younger groups. However, there were no significant differences between groups in fasting insulin, high sensitive C-reactive protein, and plasminogen activator inhibitor 1 concentrations. There were also no relationships between inflammation/ oxidative stress and the metabolic syndrome or cardiovascular disease although nonagenarians appear to be protected from oxidative damage to DNA.

Keywords Aging Cardiovascular disease . Inflammation $\cdot$ Metabolic syndrome $\cdot$ Oxidative stress

\section{I. Frisard}

Virginia Polytechnic Institute and State University,

Blacksburg, VA, USA

J. C. Rood $\cdot$ X. Fang $\cdot$ E. Ravussin $(\bowtie)$

Health and Human Performance Enhancement Division,

Pennington Biomedical Research Center,

6400 Perkins Rd,

Baton Rouge, LA 70808, USA

e-mail: ravusse@pbrc.edu

J. Su $\cdot$ D. A. Welsh

Louisiana State University Health Sciences Center, New Orleans, LA, USA

S. M. Jazwinski

Tulane University, New Orleans, LA, USA 


\section{Introduction}

The average life expectancy in the United States is 77 years, and in the western world the chance of living to 100 is 1 in 10,000 (Oeppen and Vaupel 2002; Perls et al. 2002). Individuals achieving this longevity appear to delay or even escape various age-related diseases such as type 2 diabetes mellitus (T2DM) and cardiovascular disease (CVD) that typically develop at younger ages (Perls et al. 2000). These individuals may therefore possess genetic and physiological traits that contribute to their exceptional longevity.

Cardiovascular disease is one of the leading causes of death in the westernized world (Kahn et al. 2005). The American Heart Association reports that $84 \%$ of individuals diagnosed with CVD are over the age of 65 years. The development of CVD is preceded by traits of the metabolic syndrome, a clustering of risk factors for CVD including obesity, dyslipidemia, hypertension, insulin resistance, and glucose intolerance (Ferrannini et al. 1991; World Health Organization 1999). Some individuals with exceptional longevity are seldom affected by the age-related alterations in CVD risk, although the mechanisms responsible for this younger profile are unknown (Keys et al. 1952; National Health and Nutrition Examination Surveys 1987; Ferrannini et al. 1991; Paolisso et al. 1995, 1997; Roubenoff and Hughes 2000; Ryan 2000; Perls et al. 2002; Barzilai et al. 2003).

Cardiovascular disease is tightly associated with inflammation (Ross 1999). Consistent with this



Fig. 1 Biosignatures of aging. As individuals age, cardiovascular disease (CVD) risk factors and biosignatures such as fasting insulin and C-reactive protein (CRP) increase. These markers can be used to assess biological age. Accordingly, for a given chronological age, individuals may have values indicating younger, similar, or older biological age (left panel, dotted paradigm is the recognition that, in addition to the well-recognized CVD risk factors including lipids and blood pressure, other factors, including hemostasis factors, markers of inflammation and oxidative stress, and acute phase proteins, are of importance. Circulating concentrations of these markers increase with age and are predictive of future CVD events (Kannel et al. 1987; Ridker et al. 1998a, b, 2004; Pearson et al. 2003; Devaraj et al. 2005). Assessment of such factors may help to determine an individual's "biological age" as opposed to their chronological age. Thus, individuals displaying exceptional longevity may be considered biologically younger than their chronological age and this may contribute to their extended lifespan (Fig. 1). This study tests the hypothesis that nonagenarians have levels of risk factors for CVD comparable to aged subjects and will present with less inflammation and oxidative stress.

\section{Methods}

\section{Subjects}

The subjects included in this analysis represent a subset of the Louisiana Healthy Aging Study (LHAS) funded as a program project from the National Institute on Aging (Frisard et al. 2007). In this project, extensive data were collected in three groups of individuals aged 20-34, 60-74, and $>90$ years. Subjects were excluded if they had been diagnosed



lines, shown with prediction limits). For example, an individual aged $\sim 70$ years may have values that are much lower than predicted, indicating they are younger than their chronological age. We hypothesize in this study that nonagenarians are biologically younger than their chronological age (right panel) and this contributes to their exceptional longevity 
with diabetes or had elevated fasting blood sugar $(>125 \mathrm{mg} / \mathrm{dl})$, thyroid disease, unstable CVD or mental health problems requiring drug treatment. Nonagenarians were additionally excluded if they had a heart attack or stroke in the 3 months prior to testing, severe high blood pressure, blood vessel aneurysm, taking certain medications used for myasthenia gravis or had uncontrolled asthma, an asthmalike condition or emphysema/chronic obstructive pulmonary disease (COPD). The study was approved by the Institutional Review Boards of the Pennington Biomedical Research Center and the Louisiana State University Health Sciences Center, New Orleans.

Body composition assessment

Weight was measured to $\pm 0.1 \mathrm{~kg}$ with an electronic scale (Detecto, Webb City, MO). Height was measured to $\pm 0.5 \mathrm{~cm}$ with a wall-mounted stadiometer (Holtain; Crymych, Dyfed, UK), and body mass index (BMI) was calculated as weight divided by height squared $\left(\mathrm{kg} / \mathrm{m}^{2}\right)$. Waist circumference was measured with a standard tape measure while the subject was in the standing position. Whole body composition was estimated by dual-energy X-ray absorptiometry (DXA) (Hologics, QDR 4500A, Bedford, MA).

\section{Blood pressure assessment}

Blood pressure was measured in a quiet room with minimal temperature fluctuations following a $5 \mathrm{~min}$ rest period. All measurements were taken twice (and averaged) on the participant's right arm with a manual sphygmomanometer (Baum, Coplaque, NY).

Blood and urinary analyses

Blood samples were drawn in fasting conditions for serum lipids with HDL-C measured by a Sigma Chemical (St. Louis, MO) kit whereas LDL-C was calculated using the Friedewald equation (Friedewald et al. 1972). Glucose was analyzed using a glucose oxidase electrode (Beckman Synchron CX7, Brea, $\mathrm{CA}$ ), and insulin, high sensitivity $\mathrm{C}$-reactive protein (hsCRP), and homocysteine were measured by immunoassays on a DPC 2000 (Diagnostic Product Corporation, Los Angeles, CA). The HOMA index was calculated as fasting glucose $(\mathrm{mmol} / \mathrm{L}) \times$ fasting insulin $(\mu \mathrm{U} / \mathrm{mL}) / 22.5$. Factor VII activity and fibrinogen were measured on an ACL 3000 (Brea, CA). Factor VII was expressed as percent relative to serial dilution of pooled plasma (Clauss 1957). Plasminogen activator inhibitor 1 (PAI-1) was measured by enzyme-linked immunosorbent assay (ELISA) using a kit from American Diagnostica (Stamford, CT). As indices of oxidative stress, urinary isoprostanes were measured using liquid chromatography with mass spectrometry (Davies et al. 2006), serum protein carbonyl content using a modified 2,4-dinitrophenylhydrazine (DNPH) technique (Mates et al. 2000) and DNA fragmentation by single cell gel electrophoresis (Comet and FLARE assay) (Deutsch et al. 2001). Interleukin six (IL-6) was measured by ELISA using a kit from LINCO (St Charles, MO) and run on a Luminex IS 100 (Austin, TX).

Metabolic syndrome classification

Metabolic syndrome was defined according to the Adult Treatment Panel III (ATP III) definition if at least three or more of the following criteria were present: (1) waist circumference $>102 \mathrm{~cm}$ in men or $>88 \mathrm{~cm}$ in women, (2) Triglycerides $>150 \mathrm{mg} / \mathrm{dl}$ or treatment for hypertriglyceridemia, (3) HDL cholesterol $<40 \mathrm{mg} / \mathrm{dl}$ for men or $<50 \mathrm{mg} / \mathrm{dl}$ for women or treatment for dyslipidemia, (4) Blood pressure $>130 / 85 \mathrm{mmHg}$ or documented use of antihypertensive therapy, (5) Fasting glucose concentration $>100 \mathrm{mg} / \mathrm{dl}$.

Statistical analysis

Data are presented as means \pm SEM. Data analyses were performed using SAS version 9.1.3 (SAS Institute, Cary, NC). Insulin and triglyceride were $\log$ transformed to correct for skewing but are presented using untransformed values. Age group and sex effects for subject characteristics were tested using a two-way factorial ANOVA (analysis of variance) and BMI was included as a covariate in the two-way ANOVA model when testing for the cardiovascular risk factors. The statistical significance for all multiple comparisons was adjusted with respect to the Tukey-Kramer method to control for Type I error rate. The level of significance for all statistical tests was set at $P<0.05$. Pearson correlation coefficients were used to assess relationships between risk factors for CVD, inflammatory markers, and oxidative stress. 


\section{Results}

A total of 208 participants were enrolled in the present study. The three groups will be referred to as young [20 male (M), $27 \pm 1$ years; 33 female (F), $28 \pm 1$ years], aged $(29 \mathrm{M}, 69 \pm 1$; years $29 \mathrm{~F}, 69 \pm$ 1 years) and nonagenarian $(47 \mathrm{M}, 93 \pm 0.3$ years; $50 \mathrm{~F}, 92 \pm 0.3$ years). The young group consisted of 15 white males, 24 white females, 5 black males, and 9 black females. The aged group consisted of 26 white males, 23 white females, 1 black male, 5 black females, 2 "other" males, and 1 other female. The nonagenarian group consisted of 38 white males, 47 white females, 2 black males, 1 black female, 7 other males, and 2 other females. Over the 3 years necessary to recruit the 208 volunteers described, another 177 were recruited in the program. Sixty-five nonagenarians were excluded for the presence of T2DM $(N=24)$ and/or thyroid disorders $(N=41)$ and 75 individuals chose not to come for the 7-h testing at our clinical facility. There were also 25 individuals excluded from the aged group ( 8 with T2DM, 10 with thyroid disease, and 7 unavailable for 7-h testing) and 12 individuals excluded from the young group (3 with T2DM, 2 with thyroid disease, 1 with pregnancy, and 6 unavailable for 7 -h testing). Data for hsCRP, factor VII, fibrinogen, homocysteine, and PAI-1 was available for 200, 198, 198, 199, and 196 subjects, respectively. Data for IL-6, isoprostanes, protein carbonyls, and the comet assays were available for $133,86,199$, and 189 subjects, respectively.

\section{Body composition}

Body composition characteristics of the subjects in the three age groups are displayed in Table 1. In all three age groups, males weighed significantly more than females $(P<0.001)$ and females had higher percent body fat than males $(P<0.001)$. Nonagenarians weighed significantly less than either the young or the aged group (both, $P<0.001$ ) and had a significantly lower BMI (both, $P<0.001$ ). Thirty percent of the young group, $38 \%$ of the aged group, and $8 \%$ of the nonagenarians were obese (BMI $>30$ ) whereas $30 \%, 53 \%$ and $43 \%$ were overweight (30> BMI $>25$ ), respectively.

Metabolic syndrome

Thirteen percent of the young group, $63 \%$ of the aged group, and $35 \%$ of the nonagenarians were classified
Table 1 Subject characteristics. Values are presented as means + SEM. Mean values within a row not sharing a common lower case letter are significantly different $(P<0.05)$ for pair-wise comparisons by age group and sex when interaction is significant. Non. Nonagenarian, $B M I$ body mass index, $A \times S$ age group by sex interaction

\begin{tabular}{|c|c|c|c|c|c|c|c|c|c|c|c|c|}
\hline & \multicolumn{2}{|c|}{$\begin{array}{l}\text { Young } \\
\text { (20-34 years) }\end{array}$} & \multirow{3}{*}{$\begin{array}{l}\text { Young vs } \\
\text { Aged } \\
P \text { value }\end{array}$} & \multicolumn{2}{|c|}{$\begin{array}{l}\text { Aged } \\
(60-74 \text { years })\end{array}$} & \multirow{3}{*}{$\begin{array}{l}\text { Aged vs } \\
\text { Non. } \\
P \text { value }\end{array}$} & \multicolumn{2}{|c|}{$\begin{array}{l}\text { Nonagenarian } \\
\text { (>90 years) }\end{array}$} & \multirow{3}{*}{$\begin{array}{l}\text { Young vs } \\
\text { Non } \\
P \text { value }\end{array}$} & \multicolumn{3}{|c|}{$\begin{array}{l}\text { Statistical significance of } \\
\text { difference between means }\end{array}$} \\
\hline & Men & Women & & Men & Women & & Men & Women & & $\begin{array}{l}\text { Age } \\
\text { group (A) }\end{array}$ & Sex $(S)$ & $\mathrm{A} \times \mathrm{S}$ \\
\hline & $n=20$ & $n=33$ & & $n=29$ & $n=29$ & & $n=47$ & $n=50$ & & & & \\
\hline Age (years) & $27 \pm 1$ & $28 \pm 1$ & & $69 \pm 1$ & $69 \pm 1$ & & $93 \pm 0.3$ & $92 \pm 0.3$ & & & & \\
\hline Height $(\mathrm{cm})$ & $177 \pm 1$ & $164 \pm 1$ & 0.12 & $176 \pm 1$ & $161 \pm 2$ & $<0.001 * *$ & $170 \pm 1$ & $156 \pm 1$ & $<0.001 * *$ & $<0.001 * *$ & $<0.001^{* *}$ & 0.81 \\
\hline Weight (kg) & $90 \pm 5$ & $74 \pm 4$ & 0.84 & $90 \pm 2$ & $78 \pm 3$ & $<0.001 * *$ & $72 \pm 1$ & $60 \pm 2$ & $<0.001 * *$ & $<0.001 * *$ & $<0.001 * *$ & 0.47 \\
\hline $\begin{array}{l}\text { BMI } \\
\qquad\left(\mathrm{kg} / \mathrm{m}^{2}\right)\end{array}$ & $29 \pm 2$ & $27 \pm 1$ & 0.30 & $29 \pm 1$ & $30 \pm 1$ & $<0.001 * *$ & $25 \pm 0.4$ & $25 \pm 1$ & $<0.001 * *$ & $<0.001^{* *}$ & 0.53 & 0.43 \\
\hline $\begin{array}{l}\text { Body fat } \\
(\%)\end{array}$ & $22 \pm 2 \mathrm{a}$ & $35 \pm 1 \mathrm{~b}$ & $<0.001 * *$ & $28 \pm 1 \mathrm{c}$ & $41 \pm 1 \mathrm{~d}$ & $0.02 *$ & $27 \pm 1 \mathrm{ce}$ & $35 \pm 1 \mathrm{bf}$ & $0.02 *$ & $<0.001 * *$ & $<0.001 * *$ & $0.03 *$ \\
\hline $\begin{array}{l}\text { Fat Free } \\
\text { mass }(\mathrm{kg})\end{array}$ & $69 \pm 2 \mathrm{a}$ & $47 \pm 1 \mathrm{~b}$ & 0.22 & $65 \pm 1 \mathrm{ac}$ & $46 \pm 2 \mathrm{bd}$ & $<0.001^{* *}$ & $52 \pm 1 \mathrm{e}$ & $38 \pm 1 \mathrm{f}$ & $<0.001 * *$ & $<0.001^{* *}$ & $<0.001 * *$ & $0.006^{*}$ \\
\hline $\begin{array}{l}\text { Fat mass } \\
(\mathrm{kg})\end{array}$ & $21 \pm 3$ & $27 \pm 2$ & $0.01 *$ & $25 \pm 2$ & $32 \pm 2$ & $<0.001 * *$ & $20 \pm 1$ & $22 \pm 1$ & 0.19 & $<0.001 * *$ & $<0.001 * *$ & 0.21 \\
\hline
\end{tabular}

$* P<0.05, * * P<0.001$ 
Fig. 2 Age-related differences in components of the metabolic syndrome. All three groups were significantly different from each other $(P<0.001)$. Numbers of individuals taking medications to treat hypertension and hyperlipidemia are displayed at the bottom

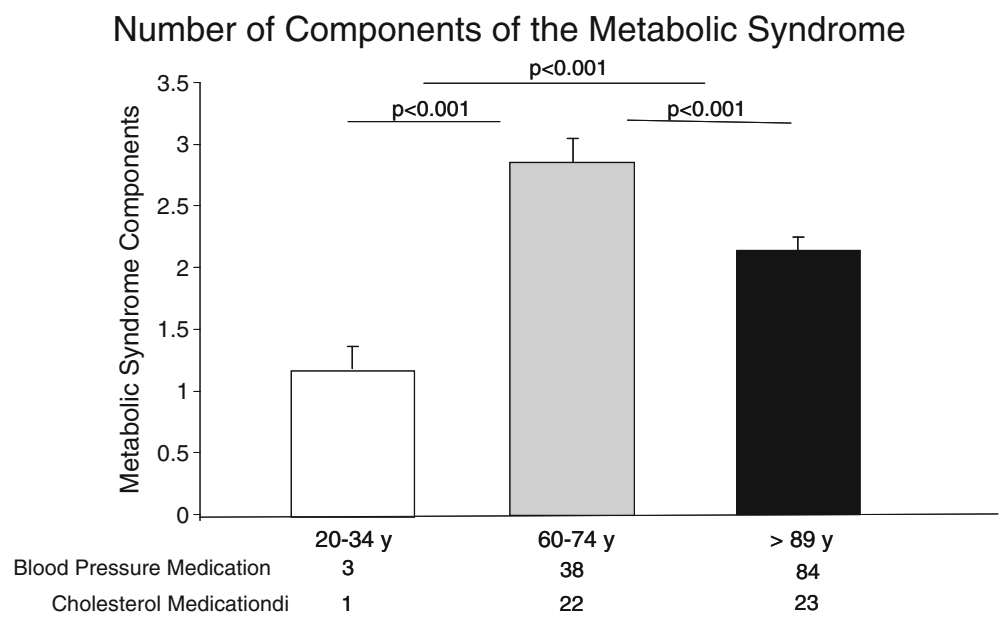

as having the metabolic syndrome. Individuals in the aged group had, on average, a significantly greater number of components of metabolic syndrome than the other two groups (Fig. 2, $P<0.001$ ). A greater number of nonagenarians were taking medication to treat blood pressure while the same number of individuals in the two older groups was taking medication to treat dyslipidemia. Average values for individual components of the metabolic syndrome for males and females are displayed in Fig. 3. As expected, men had greater waist circumference and lower HDL-C concentrations than females (both, $P<$ $0.001)$. In addition, males also had slightly higher but significant diastolic blood pressure than females $(P=$ 0.013). The two older groups had significantly larger waist circumference $(P<0.001)$, higher systolic blood pressure $(P<0.001)$, and higher fasting glucose than the young group $(P<0.001$ for aged vs young, and $P=$ 0.002 for nonagenarian vs young). Triglycerides were also significantly higher for females in the two older
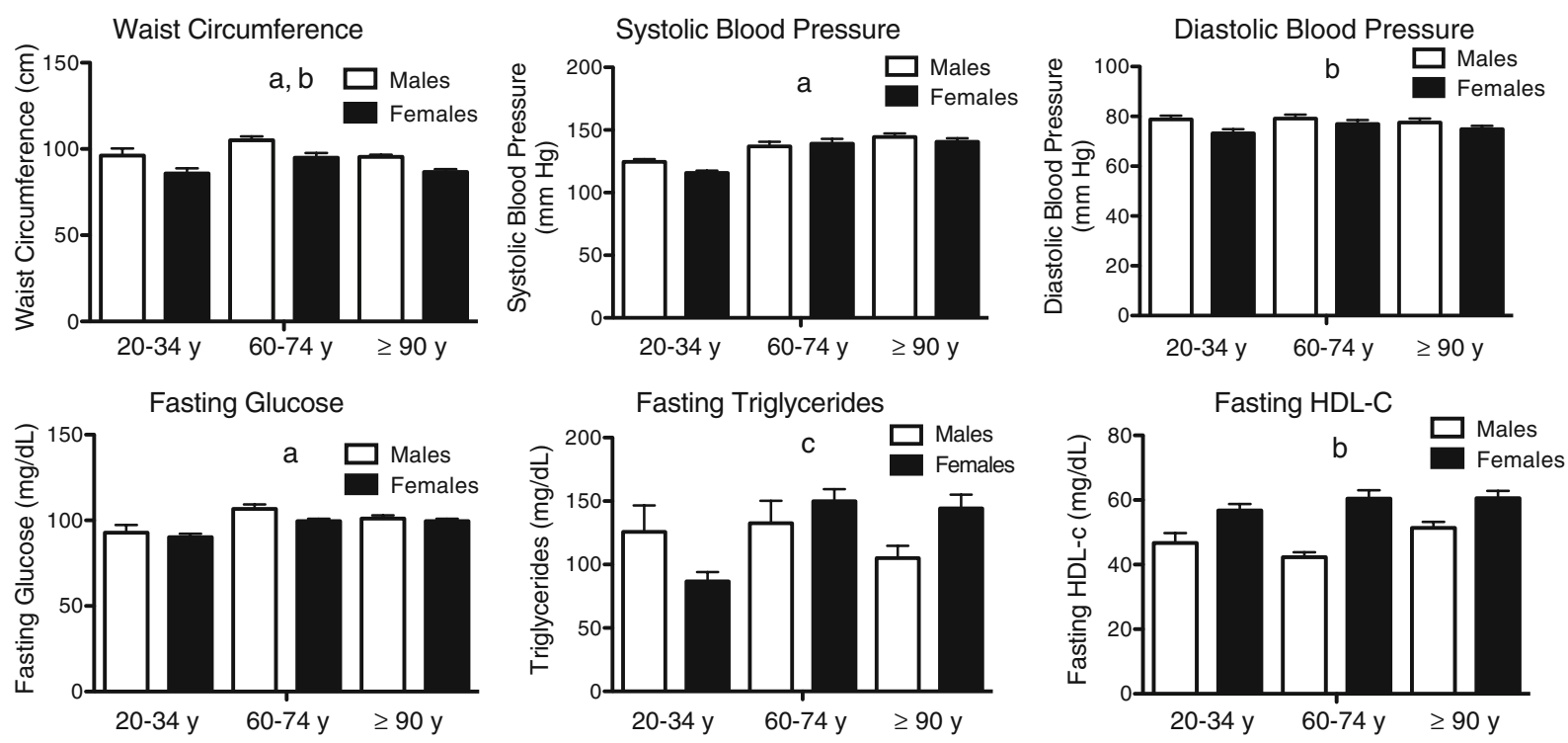

Fig. 3 Age-related differences in individual components of the metabolic syndrome separated by sex. $a$ Age group effect, $b$ sex effect, $c$ age group $\times$ sex interaction. Older and oldest females are greater than young 


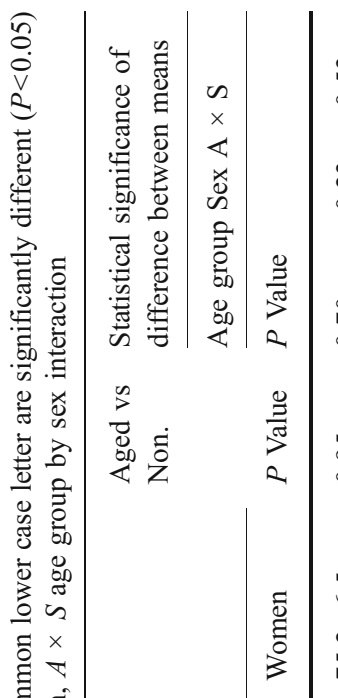

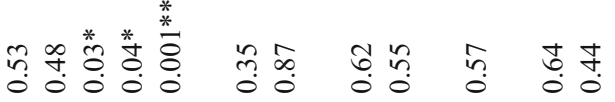
গูก





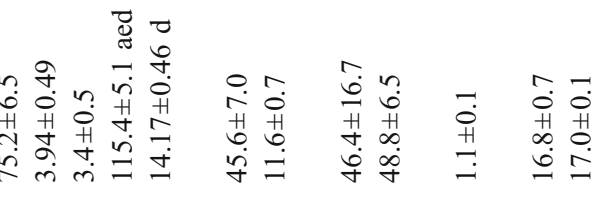
.ซี क

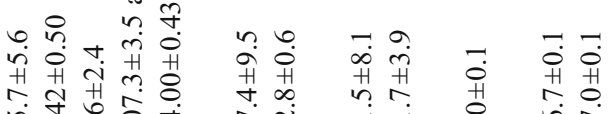
चुं

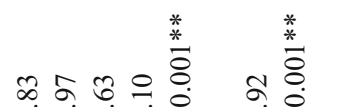

0000 ० 0 V

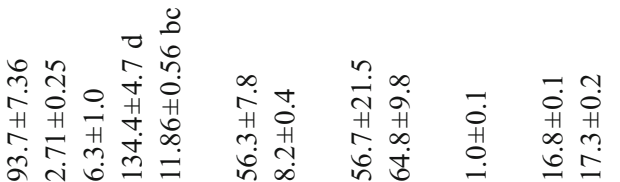
范峁

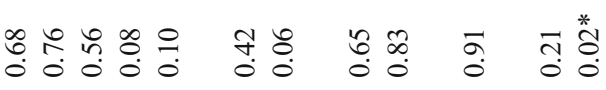

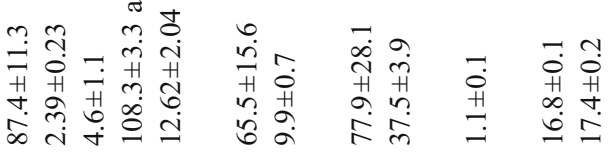
(n)







Э) 
groups compared to the young group once the data was $\log$ transformed (both $P<0.001$ ).

\section{CVD risk factors}

CVD risk factors are listed in Table 2. There were no significant differences between groups in fasting insulin, HOMA index, hsCRP, and PAI-1 concentrations nor were there any effects of sex. Males had higher homocysteine plasma concentrations than females $(P=0.008)$. Among the three age groups, the nonagenarians had higher homocysteine plasma concentrations compared to the young and the aged groups (both $P<0.001$ ). There were significant age by sex interactions for factor VII activity $(P=0.04)$ and fibrinogen $(P=0.001)$. Females of the aged group had higher factor VII activity than males in the same group $(P=0.008)$. However, factor VII activities for males among the three age groups did not differ. Females in the young group had significant lower factor VII than females in the aged group $(P=0.008)$. For fibrinogen, there were no sex differences for the aged and nonagenarian groups while the young group showed a significant difference between the sexes $(P=0.001)$. Males of the young group had less fibrinogen than either males in the aged $(P=0.002)$ or males in the nonagenarian $(P<0.001)$ groups. Females in the nonagenarian group had higher fibrinogen than females in the young $(P=0.04)$ and the aged groups $(P<0.001)$.

\section{Markers of inflammation and oxidative stress}

Markers of inflammation and oxidative stress are also listed in Table 2. There were no significant differences in urinary isoprostanes, protein carbonyls, or DNA fragmentation as measured by the comet assay between the three groups. Interestingly, females had higher urinary isoprostanes than males $(P<0.002)$. Also, the aged group had significantly higher DNA fragmentation measured by the FLARE assay than both the nonagenarians $(P=0.01)$ and the young individuals $(P=0.02)$. There was a tendency $(P=$ 0.10 ) for higher IL-6 concentrations in the aged compared to the nonagenarians although this did not reach significance. Finally, there were no significant relationships between any markers of inflammation and oxidative stress and any of the components of the metabolic syndrome.

\section{Discussion}

These results indicate that risk factors for CVD in nonagenarians are not elevated in proportion to age when compared to individuals aged 60-74. Our findings support the hypothesis that, as previously observed in centenarians, nonagenarians may be biologically young and protected from age-related disease - a factor that may contribute to their longevity. Contrary to our hypothesis, inflammation and oxidative stress were not significantly related to the components of the metabolic syndrome and/or CVD risk factors, although they were not elevated in the nonagenarians compared to the younger individuals. In fact, oxidative damage to DNA was significantly higher and IL- 6 tended to be higher in the aged group compared to the nonagenarians and the young individuals. Whether the relatively low levels of inflammation and oxidative stress in nonagenarians confer some resistance to disease remains to be shown.

The aged group had the highest prevalence of the metabolic syndrome. When comparing individual components of the metabolic syndrome in nonagenarians to the aged group, nonagenarians had mostly "better" health values except for systolic blood pressure, which was similar in the two groups. In addition to the widely recognized risk factors for CVD, there are a number of emerging biomarkers also becoming widely accepted, such hsCRP, fibrinogen, homocysteine, and PAI-1 (Devaraj et al. 2005). In our study, except for fibrinogen and homocysteine concentrations, nonagenarians had comparable levels of CVD markers to those in the younger groups. Specifically, nonagenarians had comparable CRP and PAI-1 concentrations and factor VII activities to the young individuals. PAI1 is the primary inhibitor of fibrinolysis, and factor VII is a clotting factor that promotes thrombosis. Each of these markers has been demonstrated to be predictive of future coronary events and, in fact, CRP and PAI-1 are better markers of future disease than fibrinogen or homocysteine (Kannel et al. 1987; Ridker et al. 1998b,2004; Thogersen et al. 1998). Overall, this study supports the hypothesis that nonagenarians may be protected from age-related disease.

Inflammation and oxidative stress are related directly to the progression of atherosclerosis (Wassmann and Nickenig 2003). It was expected that individuals with increased risk for CVD would also have increased inflammatory markers and higher 
oxidative damage. Only oxidative damage to DNA was found to be significantly higher in the aged group than either of the other two groups, indicating that nonagenarians may be protected from the age-related increase in DNA damage. However, the finding that inflammation and oxidative damage were not related to cardiovascular risks in the observed population therefore does not support the hypothesis that such factors are implicated in the development of the metabolic syndrome and CVD. On the contrary, the absence of increased inflammation and oxidative stress in the nonagenarians, coupled with levels of CVD markers comparable to younger individuals suggests that nonagenarians may be biologically younger than their chronological age.

Limitations of the current study include potential selection bias and/or birth cohort effects. The prevalence of obesity in the current study was 30,38 , and $8 \%$ in the young, aged, and nonagenarians groups, respectively. As such, more individuals from the younger groups may have been "unhealthier" with increased risk of disease thereby diluting differences that would have otherwise been observed. In addition, there were extensive exclusion criteria in order to prevent the confounding effects of existing disease, which may have influenced the study variables. The disadvantage to this is that we may have biased our sample towards a healthier population of older individuals. Consequently, our results should be interpreted with caution.

\section{Conclusion}

In conclusion, nonagenarians had less prevalence of the metabolic syndrome and cardiovascular risk factors, which were comparable, if not lower, to those of individuals aged $60-74$ years, indicating that their longevity may occur as a result of their biological resistance - probably genetically determined - to disease. Furthermore, the low prevalence of metabolic syndrome in the nonagenarians was not directly related to reduced inflammation and accumulation of oxidative stress.

Acknowledgments We would like to acknowledge and thank everyone working on the Louisiana Healthy Aging Study from four sites, i.e., the Pennington Biomedical Research Center, Louisiana State University in Baton Rouge, Louisiana State University Health Sciences Center in New Orleans, and the
University of Alabama in Birmingham. Most of all, we would like to thank all the participants who enrolled in the Louisiana Healthy Aging Study.

Open Access This article is distributed under the terms of the Creative Commons Attribution Noncommercial License which permits any noncommercial use, distribution, and reproduction in any medium, provided the original author(s) and source are credited.

\section{References}

American Heart Association (2005) http://www.americanheart. org/

Barzilai N, Atzmon G et al (2003) Unique lipoprotein phenotype and genotype associated with exceptional longevity. JAMA 290(15):2030-2040

Clauss A (1957) Rapid physiological coagulation method in determination of fibrinogen. Acta Haematol 17(4):237-246

Davies SS, Zackert W et al (2006) Quantification of dinor, dihydro metabolites of $\mathrm{F}(2)$-isoprostanes in urine by liquid chromatography/tandem mass spectrometry. Anal Biochem 348(2):185-191

Deutsch WA, Kukreja A et al (2001) Phenobarbital, oxazepam and Wyeth 14,643 cause DNA damage as measured by the Comet assay. Mutagenesis 16(5):439-442

Devaraj S, O'Keefe G et al (2005) Defining the proinflammatory phenotype using high sensitive C-reactive protein levels as the biomarker. J Clin Endocrinol Metab 90 (8):4549-4554

Ferrannini E, Haffner SM et al (1991) Hyperinsulinaemia: the key feature of a cardiovascular and metabolic syndrome. Diabetologia 34(6):416-422

Friedewald WT, Levy RI et al (1972) Estimation of the concentration of low-density lipoprotein cholesterol in plasma, without use of the preparative ultracentrifuge. Clin Chem 18(6):499-502

Frisard MI, Broussard A et al (2007) Aging, resting metabolic rate, and oxidative damage: results from the Louisiana Healthy Aging Study. J Gerontol A Biol Sci Med Sci 62 (7):752-759

Kahn R, Buse J et al (2005) The metabolic syndrome: time for a critical appraisal: joint statement from the American Diabetes Association and the European Association for the Study of Diabetes. Diabetes Care 28(9):2289-2304

Kannel WB, Wolf PA et al (1987) Fibrinogen and risk of cardiovascular disease. The Framingham Study. JAMA 258(9):1183-1186

Keys A, Fidanza F et al (1952) The trend of serum-cholesterol levels with age. Lancet 2(5):209-210

Mates JM, Perez-Gomez C et al (2000) Allergy to drugs: antioxidant enzymic activities, lipid peroxidation and protein oxidative damage in human blood. Cell Biochem Funct 18(2):77-84

National Health and Nutrition Examination Surveys (1987) Trends in serum cholesterol levels among US adults aged 20 to 74 years. Data from the National Health and Nutrition Examination Surveys, 1960 to 1980. National Center for Health Statistics-National Heart, Lung, and Blood Institute Collaborative Lipid Group. JAMA 257(7):937-942 
Oeppen J, Vaupel JW (2002) Demography. Broken limits to life expectancy. Science 296(5570):1029-1031

Paolisso G, Gambardella A et al (1995) Body composition, body fat distribution, and resting metabolic rate in healthy centenarians. Am J Clin Nutr 62(4):746-750

Paolisso G, Gambardella A et al (1997) Preserved antilipolytic insulin action is associated with a less atherogenic plasma lipid profile in healthy centenarians. J Am Geriatr Soc 45 (12):1504-1509

Pearson TA, Mensah GA et al (2003) Markers of inflammation and cardiovascular disease: application to clinical and public health practice: a statement for healthcare professionals from the Centers for Disease Control and Prevention and the American Heart Association. Circulation 107(3):499-511

Perls T, Terry D, Silver M et al (2000) Centenarians and the genetics of longevity. Springer, Berlin

Perls TT, Wilmoth J et al (2002) Life-long sustained mortality advantage of siblings of centenarians. Proc Natl Acad Sci USA 99(12):8442-8447

Ridker PM, Buring JE et al (1998a) Prospective study of Creactive protein and the risk of future cardiovascular events among apparently healthy women. Circulation 98 (8):731-733

Ridker PM, Glynn RJ et al (1998b) C-reactive protein adds to the predictive value of total and HDL cholesterol in determining risk of first myocardial infarction. Circulation 97(20):2007-2011

Ridker PM, Brown NJ et al (2004) Established and emerging plasma biomarkers in the prediction of first atherothrombotic events. Circulation 109(25 Suppl 1): IV6-IV19

Ross R (1999) Atherosclerosis is an inflammatory disease. Am Heart J 138(5 Pt 2):S419-S420

Roubenoff R, Hughes VA (2000) Sarcopenia: current concepts. J Gerontol A Biol Sci Med Sci 55(12):M716M724

Ryan AS (2000) Insulin resistance with aging: effects of diet and exercise. Sports Med 30(5):327-346

Thogersen AM, Jansson JH et al (1998) High plasminogen activator inhibitor and tissue plasminogen activator levels in plasma precede a first acute myocardial infarction in both men and women: evidence for the fibrinolytic system as an independent primary risk factor. Circulation 98 (21):2241-2247

Wassmann S, Nickenig G (2003) Interrelationship of free oxygen radicals and endothelial dysfunction-modulation by statins. Endothelium 10(1):23-33

World Health Organization (1999) Definition, diagnosis, and classification of diabetes mellitus and its complications: Report of a WHO Consultation. WHO, Geneva 